\title{
Effective remission of an elderly patient with refractory atopic dermatitis and foot ulcers following infusion with human umbilical cord-derived mesenchymal stem cells: A case report
}

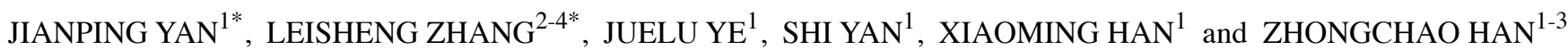 \\ ${ }^{1}$ General Outpatient Department, Jiangxi Health-Biotech Medical Development Co., Ltd.; ${ }^{2}$ Jiangxi Research Centre of \\ Stem Cell Engineering, Jiangxi Health-Biotech Stem Cell Technology Co., Ltd., Shangrao, Jiangxi 334000; \\ ${ }^{3}$ Precision Medicine Division, Health-Biotech (Tianjin) Stem Cell Research Institute Co., Ltd., \\ Tianjin 301700; ${ }^{4}$ Department of Neurosurgery, The First Affiliated Hospital of \\ Shandong First Medical University, Jinan, Shandong 250014, P.R. China
}

Received November 20, 2020; Accepted December 28, 2020

DOI: $10.3892 /$ wasj.2020.81

\begin{abstract}
Mesenchymal stem cells (MSCs) are excellent sources for use in the treatment of refractory and relapsing disorders mainly due to their hematopoietic-supporting and immunoregulatory capacities. However, their safety and effectiveness for the treatment of refractory atopic dermatitis (AD) and multiple complications in elder patients are largely unknown. Therewith, the present study describes the case of a 65-year-old female patient with AD accompanied by foot ulcers and refractory urinary tract infection. Briefly, $5 \times 10^{7}$ human umbilical cord-derived MSCs (hUC-MSCs) were intravenously infused into the body of the patient after obtaining the approval of the relevant ethics committee and the consent of the patient. The clinical and biochemical outcomes, including general symptoms, hemogram and blood constituents, medical images, together with tumour biomarkers, were observed and recorded. By conducting only one single intravenous infusion of hUC-MSCs, the AD-associated pathophysiological manifestations of the elderly patient, including an unbearable itch,
\end{abstract}

Correspondence to: Dr Leisheng Zhang or Professor Zhongchao Han, Jiangxi Research Centre of Stem Cell Engineering, Jiangxi Health-Biotech Stem Cell Technology Co., Ltd., 001 Lanjiang Road, Shangrao, Jiangxi 334000, P.R. China

E-mail: leisheng_zhang@163.com

E-mail: hanzhongchao@health-biotech.com

*Contributed equally

Abbreviations: hUC-MSCs, human umbilical cord-derived mesenchymal stem cells; AD, atopic dermatitis; hUCB-MSCs, human umbilical cord blood-derived MSCs; CEA, carcino-embryonic antigen; AFP, alpha fetoprotein; CA, carbohydrate antigen; NSE, neuron-specific enolase; SCCA, squamous cell carcinoma antigen

Key words: human umbilical cord-derived mesenchymal stem cells, atopic dermatitis, foot ulcers, urinary tract infection diffuse erythema and eczema, and in particular, inflammatory foot ulcers and refractory urinary infection were completely attenuated. Simultaneously, the outcomes of the accompanied complications were effectively improved, whereas no recrudesce or adverse reactions were observed in the 14-month follow-up visit. The findings of the present study suggested that the administration of hUC-MSCs was safe and effective for restricting the symptoms and of the patient preventing associated complications. Thus, the use of hUC-MSCs may prove to be a promising treatment option for $\mathrm{AD}$ and foot ulcer management, even for elderly patients with multiple underlying conditions or complications.

\section{Introduction}

Atopic dermatitis (AD), a heterogeneous skin lesion with an increasing prevalence worldwide, is recognized as the most common chronic and relapsing inflammatory disorder among infants, children and even adults of all races, particularly among families with a history of related allergic diseases $(1,2)$. Overall, AD can be divided into the intrinsic and extrinsic subtypes, which approximately comprises up 20 and $80 \%$ of patients, respectively (3). The disease has been reported to have a complex causality due to a series of immunological, genetic and environmental elements, with resultant comorbidities, such as respiratory diseases, food allergies, inflammatory skin infections and relative autoimmune disorders $(1,4)$. As previously reported by Kim et al, the curative efficacy of the current clinical regimens against AD is relatively insufficient, along with marked ambiguity and side-effects (5). Moreover, complications, including xerosis, pruritus and eczematous skin ulcers, further complicate the exploration of the pathogenesis and management of $\mathrm{AD}(4,6)$. Thus, considering the increasing evidence that AD has caused a tremendous burden upon patients and their caregivers, there is an urgent need for the establishment of a more systemic therapeutic strategy with which to eliminate the complex pathophysiology together with the accompanied non-allergic and allergic comorbidities $(2,7)$. 
Mesenchymal stem cells (MSCs) are immunomodulating cell populations with static adherence and a typically fusiform morphology, with a high-level expression of mesenchymal-associated surface markers, and a multi-lineage differentiation capacity towards adipocytes, osteoblasts and chondroblasts (8). For decades, numerous fundamental research and clinical applications have collectively demonstrated the vast prospects of the use of MSCs in regenerative medicine and the coordinate contributions to the physiological and pathological microenvironment (8-10). For instance, researchers have extensively investigated the cultivate prospects of MSCs in a variety of refractory and relapsing diseases, such as the sports-related injuries (e.g., meniscal injuries) and osteoarthritis $(11,12)$, nervous system disorders (e.g., Alzheimer's disease and spinal cord injuries) $(13,14)$, digestive disorders (e.g., acute-on-chronic liver failure and fulminant hepatic disease) $(15,16)$, endocrine disorders (e.g., diabetes mellitus and related complications) $(17,18)$, reproductive disorders (e.g. premature ovarian failure and intrauterine adhesions) $(19,20)$, haematological disorders (e.g., aplastic anaemia and acute myelogenous leukaemia) $(21,22)$, and in particular, immunodysregulation (graft-vs.-host disease and Crohn's disease) $(23,24)$. Kim et al reported that the first-in-class clinical trial upon AD administration with human umbilical cord blood-derived MSCs (hUCB-MSCs), even though hUCB-MSC infusion was well tolerated without noteworthy adverse events; yet, the efficiency together with the crucial effects did not yield statistical significance (5). Above all, the detailed effects of MSCs upon AD-associated skin ulcers and vital signs of elderly patient $>60$ years of age remain unexplored.

The present study describes the case of a 65-year-old female patient with typical symptoms of severe AD, including unbearable pruritus, xeroderma and systemic eczema plaques with refractory foot skin ulcer. With the aid of hormone and other drug treatments, the pruritus of the elderly patient was transiently attenuated, but relapsed quickly, whereas the other complications did not exhibit a visible improvement. Thus, with the consent of the patient and approval by the relevant ethics committee, $5 \times 10^{7}$ human umbilical cord-derived MSCs (hUC-MSCs) were administered into the body of the patient via intravenous infusion. Notably, the aforementioned symptoms of AD and multiple complications of the patient were completely eliminated without visible side-effects upon examination of systematic vital signs, and in particular, the severe foot ulcers in both feet had subsided. Taken together, the findings of the present study confirm the safety and efficacy of the proof-of-concept use of hUC-MSCs for the treatment of $\mathrm{AD}$. In addition, the present study highlights the prospects of the use of hUC-MSCs for elderly patients with refractory AD-associated severe skin ulcers.

\section{Case report}

General description of the patient. In March, 2019, the 65-year-old female patient in question was diagnosed with influenza and a cough, when then subsided, apart from the occasional itch and sporadic eczema spots on the left foot. The patient was treated by an oral administration of a routine dose of levofloxacin according to the drug instructions as suggested by the doctors at the General Outpatient Department,
People's Hospital of Shangrao Economic and Technological Development Zone, Jiangxi Health-Biotech Medical Development Co., Ltd. However, in the middle of April, the area of the original eczema began to gradually increase from $1.0 \times 1.5$ to $2.5 \times 5.5 \mathrm{~cm}$, and the local skin turned purple and was accompanied by a persistent itch. After 1 month, a clinical examination revealed a urinary tract infection in the patient. However, a significant remission in symptoms was observed following the administration of norfloxacin (for eczema) for 3 days. Even though the urinary tract infection was temporarily eliminated with the aid of levofloxacin treatment, yet a secondary infection was detected and this became more frequent and severe by mid-July. For the purpose of alleviating the refractory infection and the accompanied erythema in the body, an oral cefixime-based remedy was conducted for 2 weeks. Unexpectedly, the erythema in the double lower limbs diffused and increased gradually, and spread upward from the crus without signs of subsiding at all (Fig. 1A). In addition, ulcerations began to form on the feet and these expanded into the epidermis and dermis (Fig. 1B).

hUC-MSC infusion and safety outcomes. On the basis of the aforementioned information, the clinical expert panel consist of the doctors at the General Outpatient Department, People's Hospital of Shangrao Economic and Technological Development Zone, Jiangxi Health-Biotech Medical Development Co., Ltd. reached the following consensus: The elderly patient was diagnosed with refractory AD accompanied by pruritus, diffuse erythema in the body, severe skin ulcers on the feet and the incorporated urinary tract infection (Fig. 1A and B, and Table SI). Having considered the inefficacious and even cumulative outcomes of the traditionally comprehensive treatment, the patient then received an intravenous administration of $5 \times 10^{7}$ clinical grade hUC-MSCs (Jiangxi Research Centre of Stem Cell Engineering, Jiangxi Health-Biotech Stem Cell Technology Co. Ltd.; product lot no. 201909JF03) via a sterile blood transfusion needle at a rate of 45 drops per min on September 15th, 2020 in $100 \mathrm{ml}$ $0.9 \%$ saline after obtaining the ethical approval of the Ethics Committee of People's Hospital of Shangrao Economic and Technological Development Zone, Jiangxi Health-Biotech Medical Development Co., Ltd. and the informed consent of the patient. During the transfusion process, no acute adverse effects or drug-related events, such as infusion-related or allergic reactions (e.g., polypnea, slight trembling, or instantaneous low fever) were observed in the patient with AD. Subsequently, no delayed hypersensitivity, secondary infection, or life-threatening events were observed within the 14-month period of observation as well.

Complete remission of the patient with $A D$ administered the $h U C$-MSCs. To assess the therapeutic effects of the hUC-MSC administration, the clinical symptoms of the patient with AD were initially evaluated. Generally, the size and crimson colour of the diffuse erythema were collectively and gradually alleviated within the first 10 days and eventually disappeared after 1 month, which indicated the effectiveness of the infusion of MSCs upon AD-associated eczema. Moreover, the unbearable itch and refractory urinary tract infection were also alleviated from the patient with AD. Notably, during the 
A
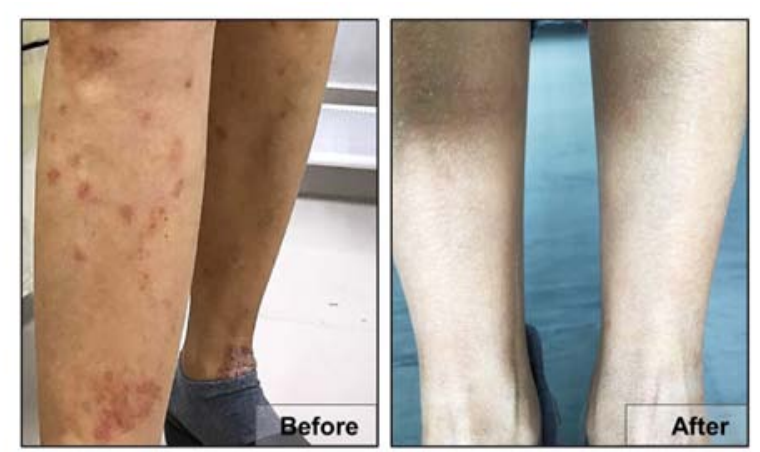

B

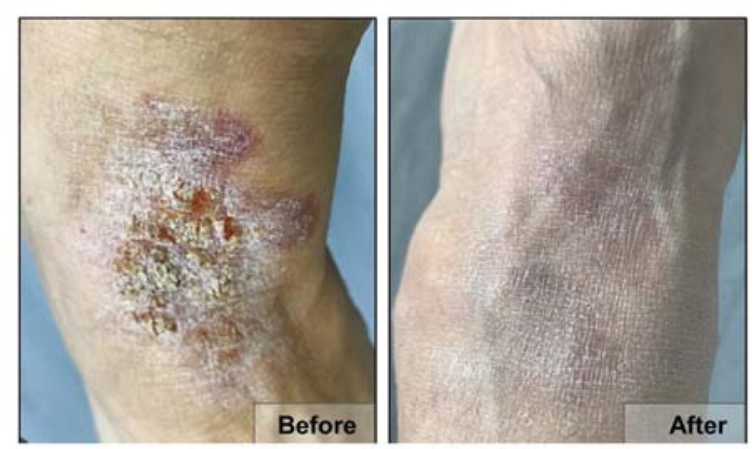

Figure 1. The pathophysiological examination of the patient with AD (A) Pathophysiological morphology of eczema plaque in the lower limbs before (left panel) and after (right panel) the hUC-MSC administration. (B) Pathophysiological morphology of refractory ulcers in the left foot before (left panel) and after (right panel) the hUC-MSC administration. AD, atopic dermatitis; hUC-MSC, human umbilical cord-derived mesenchymal stem cells.

14-month follow-up visits, no recrudescent AD-associated pathophysiological events, including itch and erythema, and in particular ulcers on the foot, were observed in the body of the elderly patient (Fig. 1A). Additionally, with only a single hUC-MSC administration, the patient with AD was satisfied with the treatment effects (with the smooth skin) and felt well in body and mind as well, which collectively revealed the improvement of the quality of life (Fig. 1B).

Apart from the subjective and objective changes in the general physical examination of the patient, multifaceted assessments, including vital signs, and detailed clinical and laboratory examinations were collectively conducted upon the patient with AD. Firstly, from the routine urine test and the microscopic examination of leucocytes (R-WBC), a marked decline in the numbers of leucocytes was observed, and a decrease was also observed the content of nitrite in the urine (Tables SI and SII). Secondly, according to blood cell test results of the patient, both blood cell components (e.g., neutrophils, lymphocytes and granulocytes) and biochemical indexes (e.g., bilirubin, alanine aminotransferase, cholesterol and high-density lipoprotein cholesterol) were within the normal ranges, without anomalous changes before treatment and after complete recovery, while the contents of total cholesterol and low-density lipoprotein cholesterol were slightly increased (Tables SI and SII). In addition, following MSC treatment, the dysregulated other blood parameters, such as Casson viscosity, whole blood shear rate 50 and 100 , and erythrocyte sedimentation rate were also ameliorated (Tables SI and SII). Above all, as shown by the test results, none of the well-known cancer-associated parameters with abnormal elevations were observed in the patient with $\mathrm{AD}$, including carcinoembryonic antigen (CEA), alpha fetoprotein (AFP), carbohydrate antigens (e.g., CA-125, CA-133, CA-199, CA-242 and CA-724), neuron-specific enolase (NSE), cytokeratin 19 (CK-19) and squamous cell carcinoma antigen (SCCA) (Tables SI and SII).

\section{Discussion}

State-of-the-art renewal has enlightened the promising prospects of MSCs in the management of refractory and relapsing disorders. This is mainly attribute to their unique hematopoietic-supporting and immunoregulatory properties $(21,25,26)$. However, their potential application in elderly patients with refractory allergic dermatitis with multiple complications are largely unknown. The present study describes the case of a 65-year-old female patient with AD accompanied by an unbearable itch, extensive eczema, severe foot ulcers, urinary tract infection, and in particular, resistance to conventional drug therapy. With the aid of only one single systemic hUC-MSC injection, the major symptoms of AD were effectively relieved within 10 days following the MSC administration. Finally, a positive outcome without palindromia was observed with the patient during the 14-month follow-up visit, including the significant elimination of pathophysiological manifestations and persistent improvement in the quality of life.

The pathogenesis of AD typically manifests in epidermal barrier disruption, dysbiosis of the skin microbiota and the overactivation of the helper T lymphocyte subpopulations (Th1, Th2, Th17 and Th22), as well as increased eosinophils and IgE in blood $(3,27)$. However, the detailed underlying mechanisms, as well as the absolute magnitude of the risks of AD remain largely unknown $(2,3,27)$. For instance, the impact of gene-environment interactions upon epidermal barrier destruction and the resultant variations in clinical presentations have not yet been clearly demonstrated (5). In addition, AD is considered to be an initiating factor for other atopic disorders, including food allergies, allergic asthma and rhinitis, which can continue for a long period of time, maintaining a relapsing-remitting status in affected patients (6). In general, the systematic and precise clarification of $\mathrm{AD}$-associated epidemiologic features, clinical phenotypes, genetic subtype, clinical remission and the underlying pathogenic mechanisms are not yet fully understood $(5,27)$. As a consequence, even though current treatment modalities, including pharmacological-associated interventions alone or in combination with non-pharmacological strategies may be able to relieve pain in patients with moderate to severe AD, their effectiveness is not satisfactory due to the complexity and indeterminacy of the underlying pathogenesis. For example, a recent advancement has been made in the monoclonal antibody (mAb), dupilumab, which has exhibited satisfactory effectiveness against $\mathrm{AD}$ with a remission rate of $85 \%$ via a subcutaneous administration (11 times) by blocking IL-3 and IL-13 (28). However, in spite of its effectiveness, the accompanied adverse reactions, inconvenience and unexpected clinical outcomes (e.g., headache, injection-site reaction and conjunctivitis), would largely hinder its use in the rational and effective application in patients with $\mathrm{AD}(29,30)$.

Distinguishing from the traditional or late-model drug-based strategies, MSC-based cytotherapy has been proven 
to be safe and effective against multiple disorders associated with immune abnormalities (31-34). Since the first separation and identification in the 1960s, MSCs have been successfully isolated from adult tissues, perinatal tissues and even derived from human pluripotent stem cells (35-37). Of these, the natural bone marrow- and umbilical cord-segregated MSCs are acknowledged with the most popular and proliferative characteristics, respectively $(21,23,24)$. MSCs function mainly via cytokine paracrine mechanisms [e.g., prostaglandin E2 (PGE2) and transforming growth factor (TGF)- $\beta 1$ ], the inhibition of inflammatory factor production [e.g., interferon (IFN)- $\gamma$ and tumour necrosis factor (TNF)- $\alpha$, the recruitment and regulation of other cell constituents (e.g., mast cells, T lymphocytes) and direct differentiation, together with providing a favourable microenvironment (9,38-40). As for the management of AD, by administering a high-dose of hUCB-MSCs $\left(5 \times 10^{7}\right)$ via local subcutaneous injection, Kim et al reported few adverse events and a certain improvement of patients with $\mathrm{AD}$ with an overall response rate of 55\% at week 12 (40).

However, the systemic infusion and long-term effects of MSCs against AD remain unknown. To the best of our knowledge, the present study demonstrates for the first time that hUC-MSCs were effective in the treatment of a patient with AD with other complications, including foot ulcers and a urinary tract infection, leading to a full, rather than partial remission, via a single and convenient intravenous injection. In particular, the severe ulcers and refractory urinary tract infection in the elderly patient with AD were completely eliminated without recrudesce during the 14-month follow-up visit. Simultaneously, according to the systematic clinical and biochemical examinations, and in particular, the tumour-related indicators, the consistent safety and continuous efficacy of MSC-based cytotherapy for AD was confirmed. Nevertheless, more patients with AD need to be enrolled and examined in order to evaluate the reliability and validity of hUC-MSC infusion via intravenous injection. Overall, the data presented herein provide a paradigm and prospect for further investigations towards AD treatment, and may help to predominantly ameliorate the refractory disorder in the future.

\section{Acknowledgements}

The authors would like to thank Health-Biotech (Tianjin) Stem Cell Research Institute Co., Ltd. for their kind support.

\section{Funding}

The present study was supported by the National Science and Technology Major Projects of China (2014ZX09508002-003), the project funded by the China Postdoctoral Science Foundation (2019M661033), the project Youth Fund supported by Shandong Provincial Natural Science Foundation (ZR2020QC097), the Key project funded by the Department of Science and Technology of Shangrao City (2020XGFY05), the Key project funded by the Department of Science and Technology of Shangrao City (2021, to JYa), the Jiangxi Key New Product Incubation Program funded by the Technical Innovation Guidance Program of Shangrao city (2021, to LZ) and the Natural Science Foundation of Fujian Province (2020J01649).

\section{Availability of data and materials}

The data used to support the findings of the present study are included in the article. Additional data related to the study are available from the corresponding author. In addition, a trial registration has been made as follows: Clinical Classification, Epidemiological Investigation and Mechanism of Skin Barrier Dysfunction of Hand Eczema in Outpatient Clinics of Hospitals in China, ChiCTR1800018943. Registered October 17, 2018 (http://www.chictr.org.cn/showproj.aspx?proj=31989; prospectively registered); and 'Human mesenchymal stem cell in the treatment of wounds in partial-thickness skin donor site in burn patients: A randomized controlled trial', ChiCTR2000038275. Registered September 15, 2020 (http://www.chictr.org. $\mathrm{cn} /$ showproj.aspx?proj=56515; prospectively registered).

\section{Authors' contributions}

JYa was involved in the collection and assembly of data and in manuscript writing. $\mathrm{XH}, \mathrm{JYe}$ and SY were involved in the collection and assembly of data. $\mathrm{LZ}$ and $\mathrm{ZH}$ were involved in the conception and design of the study, and in manuscript writing and revision. All authors read and approved the final manuscript.

\section{Ethics approval and consent to participate}

The treatment of the patient followed the internationally recognized guidelines and the principles of the Declaration of Helsinki. Ethical approval for the research was signed by the Ethics Committee of Jiangxi Health-Biotech Development Co., Ltd., China (approval no. EC-2019-01). The patient signed an informed consent to the publication of her case report.

\section{Patient consent for publication}

The patient signed an informed consent to the publication of her case report.

\section{Competing interests}

The authors declare that they have no competing interests.

\section{References}

1. Torres T, Ferreira EO, Gonçalo M, Mendes-Bastos P, Selores M and Filipe P: Update on atopic dermatitis. Acta Med Port 32: 606-613, 2019

2. Cabanillas B, Brehler AC and Novak N: Atopic dermatitis phenotypes and the need for personalized medicine. Curr Opin Allergy Clin Immunol 17: 309-315, 2017.

3. Suárez-Fariñas M, Dhingra N, Gittler J, Shemer A, Cardinale I, de Guzman Strong C, Krueger JG and Guttman-Yassky E: Intrinsic atopic dermatitis shows similar TH2 and higher TH17 immune activation compared with extrinsic atopic dermatitis. J Allergy Clin Immunol 132: 361-370, 2013.

4. Leung DY, Nicklas RA, Li JT, Bernstein IL, Blessing-Moore J, Boguniewicz M, Chapman JA, Khan DA, Lang D, Lee RE, et al: Disease management of atopic dermatitis: An updated practice parameter. Joint Task Force on Practice Parameters. Ann Allergy Asthma Immunol 93 (3 Suppl 2): S1-S21, 2004.

5. Kim HS, Lee JH, Roh KH, Jun HJ, Kang KS and Kim TY: Clinical trial of human umbilical cord blood-derived stem cells for the treatment of moderate-to-severe atopic dermatitis: Phase I/IIa studies. Stem Cells 35: 248-255, 2017. 
6. Schneider L, Tilles S,Lio P,Boguniewicz M, Beck L,LeBovidge J, Novak N, Bernstein D, Blessing-Moore J, Khan D, et al: Atopic dermatitis: A practice parameter update 2012. J Allergy Clin Immunol 131: 295-299.e1-27, 2013.

7. Silverberg NB and Duran-McKinster C: Special considerations for therapy of pediatric atopic dermatitis. Dermatol Clin 35: 351-363, 2017.

8. Huo J, Zhang L, Ren X, Li C, Li X, Dong P, Zheng X, Huang J, Shao Y, Ge M, et al: Multifaceted characterization of the signatures and efficacy of mesenchymal stem/stromal cells in acquired aplastic anemia. Stem Cell Res Ther 11: 59, 2020.

9. Shi Y, Wang Y, Li Q, Liu K, Hou J, Shao C and Wang Y: Immunoregulatory mechanisms of mesenchymal stem and stromal cells in inflammatory diseases. Nat Rev Nephrol 14 493-507, 2018.

10. Menasche P: Cell therapy trials for heart regeneration-lessons learned and future directions. Nat Rev Cardiol 15: 659-671, 2018

11. Matas J, Orrego M, Amenabar D, Infante C, Tapia-Limonchi R, Cadiz MI, Alcayaga-Miranda F, González PL, Muse E, Khoury M, et al: Umbilical cord-derived mesenchymal stromal cells (MSCs) for knee osteoarthritis: Repeated MSC dosing is superior to a single MSC dose and to hyaluronic acid in a controlled randomized phase I/II trial. Stem Cells Transl Med 8: 215-224, 2019.

12. Hidalgo Perea S, Lyons LP, Nishimuta JF, Weinberg JB and McNulty AL: Evaluation of culture conditions for in vitro meniscus repair model systems using bone marrow-derived mesenchymal stem cells. Connect Tissue Res 61: 322-337, 2020.

13. Reza-Zaldivar EE, Hernández-Sapiéns MA, Minjarez B, Gutiérrez-Mercado YK, Márquez-Aguirre ÁL and Canales-Aguirre AA: Potential effects of MSC-derived exosomes in neuroplasticity in Alzheimer's disease. Front Cell Neurosci 12: 317,2018

14. Wu GH, Shi HJ, Che MT, Huang MY, Wei QS, Feng B, Ma YH, Wang LJ, Jiang B, Wang YQ, et al: Recovery of paralyzed limb motor function in canine with complete spinal cord injury following implantation of MSC-derived neural network tissue. Biomaterials 181: 15-34, 2018.

15. Huang B, Cheng X, Wang H, Huang W, la Ga Hu Z, Wang D, Zhang K, Zhang H, Xue Z, Da Y, et al: Mesenchymal stem cells and their secreted molecules predominantly ameliorate fulminant hepatic failure and chronic liver fibrosis in mice respectively. J Transl Med 14: 45, 2016.

16. Lin BL, Chen JF, Qiu WH, Wang KW, Xie DY, Chen XY, Liu QL Peng L, Li JG, Mei YY, et al: Allogeneic bone marrow-derived mesenchymal stromal cells for hepatitis B virus-related acute-on-chronic liver failure: A randomized controlled trial. Hepatology 66: 209-219, 2017.

17. Wang L, Liu T, Liang R, Wang G, Liu Y, Zou J, Liu N, Zhang B, Liu Y, Ding X, et al: Mesenchymal stem cells ameliorate beta cell dysfunction of human type 2 diabetic islets by reversing beta cell dedifferentiation. EBioMedicine 51: 102615, 2020.

18. Li B, Luan S, Chen J, Zhou Y, Wang T, Li Z, Fu Y, Zhai A and Bi C: The MSC-derived exosomal lncRNA H19 promotes wound healing in diabetic foot ulcers by upregulating PTEN via MicroRNA-152-3p. Mol Ther Nucleic Acidrs 19: 814-826, 2020.

19. Ding L, Yan G, Wang B, Xu L, Gu Y, Ru T, Cui X, Lei L, Liu J, Sheng X, et al: Transplantation of UC-MSCs on collagen scaffold activates follicles in dormant ovaries of POF patients with long history of infertility. Sci China Life Sci 61: 1554-1565, 2018.

20. Liu F, Hu S, Yang H, Li Z, Huang K, Su T, Wang S and Cheng K: Hyaluronic acid hydrogel integrated with mesenchymal stem cell-secretome to treat endometrial injury in a rat model of Asherman's syndrome. Adv Healthc Mater 8: e1900411, 2019.

21. Wei Y, Zhang L, Chi Y, Ren X, Gao Y, Song B, Li C, Han Z, Zhang L and Han Z: High-efficient generation of VCAM-1(+) mesenchymal stem cells with multidimensional superiorities in signatures and efficacy on aplastic anaemia mice. Cell Prolif: e12862, 2020.

22. Song K, Li W and Li M: Acute promyelocytic leukemia following autologous bone marrow-derived mesenchymal stem cell transplantation for traumatic brain injury: A case report. Oncol Lett 10: 2905-2908, 2015.

23. Zhao Q, Zhang L, Wei Y, Yu H, Zou L, Huo J, Yang H, Song B, Wei T, Wu D, et al: Systematic comparison of hUC-MSCs at various passages reveals the variations of signatures and therapeutic effect on acute graft-versus-host disease. Stem Cell Res Ther 10: 354, 2019.
24. Hou H, Zhang L, Duan L, Liu Y, Han Z, Li Z and Cao X Spatio-temporal metabolokinetics and efficacy of human placenta-derived mesenchymal stem/stromal cells on mice with refractory Crohn's-like enterocutaneous fistula. Stem Cell Rev Rep 16: 1292-1304, 2020.

25. Golchin A, Seyedjafari E and Ardeshirylajimi A: Mesenchymal Stem Cell Therapy for COVID-19: Present or Future. Stem Cell Rev Rep 16: 427-433, 2020.

26. Kabat M, Bobkov I, Kumar S and Grumet M: Trends in mesenchymal stem cell clinical trials 2004-2018: Is efficacy optimal in a narrow dose range? Stem Cells Transl Med 9: 17-27, 2019.

27. Guttman-Yassky E, Nograles KE and Krueger JG: Contrasting pathogenesis of atopic dermatitis and psoriasis-part II: Immune cell subsets and therapeutic concepts. J Allergy Clin Immunol 127: 1420-1432, 2011.

28. Beck LA, Thaçi D, Hamilton JD, Graham NM, Bieber T, Rocklin R, Ming JE, Ren H, Kao R, Simpson E, et al: Dupilumab treatment in adults with moderate-to-severe atopic dermatitis. $\mathrm{N}$ Engl J Med 371: 130-139, 2014

29. Ou Z, Chen C, Chen A, Yang Y and Zhou W: Adverse events of Dupilumab in adults with moderate-to-severe atopic dermatitis: A meta-analysis. Int Immunopharmacol 54: 303-310, 2018.

30. Blauvelt A, de Bruin-Weller M, Gooderham M, Cather JC, Weisman J, Pariser D, Simpson EL, Papp KA, Hong HC, Rubel D, et al: Long-term management of moderate-to-severe atopic dermatitis with dupilumab and concomitant topical corticosteroids (LIBERTY AD CHRONOS): A 1-year, randomised, double-blinded, placebo-controlled, phase 3 trial. Lancet 389: 2287-2303, 2017.

31. Cho J, D'Antuono M, Glicksman M, Wang J and Jonklaas J: A review of clinical trials: Mesenchymal stem cell transplant therapy in type 1 and type 2 diabetes mellitus. Am J Stem Cells 7: 82-93,2018.

32. Gonzaga VF, Wenceslau CV, Lisboa GS, Frare EO and Kerkis I: Mesenchymal stem cell benefits observed in bone marrow failure and acquired aplastic anemia. Stem Cells Int 2017: 8076529, 2017.

33. Dalal J, Gandy K and Domen J: Role of mesenchymal stem cell therapy in Crohn's disease. Pediatr Res 71: 445-451, 2012.

34. Pourgholaminejad A, Aghdami N, Baharvand $\mathrm{H}$ and Moazzeni SM: The effect of pro-inflammatory cytokines on immunophenotype, differentiation capacity and immunomodulatory functions of human mesenchymal stem cells. Cytokine 85: 51-60, 2016

35. Wei Y, Hou H, Zhang L, Zhao N, Li C, Huo J, Liu Y, Zhang W, Li Z, Liu D, et al: JNKi- and DAC-programmed mesenchymal stem/stromal cells from hESCs facilitate hematopoiesis and alleviate hind limb ischemia. Stem Cell Res Ther 10: 186, 2019.

36. Yao J, Chen N, Wang X, Zhang L, Huo J, Chi Y, Li Z and Han Z: Human supernumerary teeth-derived apical papillary Stem Cells possess preferable characteristics and efficacy on hepatic fibrosis in mice. Stem Cells Int 2020: 6489396, 2020.

37. Du W, Li X, Chi Y, Ma F, Li Z, Yang S, Song B, Cui J, Ma T, Li J, et al: VCAM-1 ${ }^{+}$placenta chorionic villi-derived mesenchymal stem cells display potent pro-angiogenic activity. Stem Cell Res Ther 7: 49, 2016.

38. Fan XL, Zhang Y, Li X and Fu QL: Mechanisms underlying the protective effects of mesenchymal stem cell-based therapy. Cell Mol Life Sci 77: 2771-2794, 2020.

39. Kfoury Y and Scadden DT: Mesenchymal cell contributions to the stem cell niche. Cell Stem Cell 16: 239-253, 2015.

40. Kim HS, Yun JW, Shin TH, Lee SH, Lee BC, Yu KR, Seo Y, Lee S, Kang TW, Choi SW, et al: Human umbilical cord blood mesenchymal stem cell-derived PGE2 and TGF- $\beta 1$ alleviate atopic dermatitis by reducing mast cell degranulation. Stem Cells 33: 1254-1266, 2015.

This work is licensed under a Creative Commons Attribution-NonCommercial-NoDerivatives 4.0 International (CC BY-NC-ND 4.0) License. 